TAIWANESE JOURNAL OF MATHEMATICS

Vol. 17, No. 1, pp. 1-13, February 2013

DOI: $10.11650 /$ tjm.17.2013.1954

This paper is available online at http://journal.taiwanmathsoc.org.tw

\title{
ON SHARP LOWER BOUND OF THE GAP FOR THE FIRST TWO EIGENVALUES IN THE SCHRÖDINGER OPERATOR
}

Yue He

\begin{abstract}
. [8] is a deep study in the sharp lower bound estimate of the gap for the first two eigenvalues in Schrödinger operator on a smooth bounded convex domain in $\mathbb{R}^{n}$. In this paper we give another simple proof of the main result in [8]. Although the methods used in here due to [8] on the whole, to some extent we deal with the singularity of some function and also simplify greatly calculation in [8].
\end{abstract}

\section{INTRODUCTION}

The study in the estimates of the eigenvalues has a long history. Meanwhile, there are many works in this field. Among these works, Li-Yau's results (e.g., [2-6]) and Zhong's results (e.g., [7, 8]) are all very well known. In 1979, the maximum principle method was used by Li [2] in proving eigenvalue estimates for compact manifolds. To be more precise, the maximum principle is used to deduce the gradient estimate on the eigenfunction, and then the eigenvalue estimates is obtained via the above gradient estimate. This method was then refined and used by many authors ([3, 7], etc) for obtaining sharp eigenvalue estimates.

Now we state some results on the lower bound estimate of the gap for the first two eigenvalues in Schrodinger operator on a smooth bounded convex domain in $\mathbb{R}^{n}$.

In 1985, Yau and others [6] use the method of the gradient estimate to deduce estimates on $\lambda_{2}-\lambda_{1}$. one of the main results in [6] is the following theorem.

Theorem 1.1. (Singer-Wong-Yau-Yau). Let $\Omega$ be a smooth strictly convex bounded domain in $\mathbb{R}^{n}$ and $W: \bar{\Omega} \mapsto \mathbb{R}$ a nonnegative convex function. Suppose $\lambda_{1}$ and $\lambda_{2}$ are

Received April 23, 2012, accepted May 2, 2012.

Communicated by Shu-Cheng Chang.

2010 Mathematics Subject Classification: Primary 58G25, 58G11, 35J10, 35P05, 53C20.

Key words and phrases: Schrodinger operator, The gap of the first two eigenvalues, Second fundamental form, Strictly convex domain, The diameter of domain.

Partially supported by NNSF grant of P. R. China, No. 11171158. 
the first and second nonzero eigenvalues of (2.1), then the following pinch inequality holds

$$
\frac{\pi^{2}}{4 d^{2}} \leqslant \lambda_{2}-\lambda_{1} \leqslant \frac{4 n \pi^{2}}{D^{2}}+\frac{4(M-m)}{n},
$$

where $d$ is the diameter of $\Omega, D=$ the diameter of the largest inscribed ball in $\Omega$,

$$
M=\sup _{\bar{\Omega}} W \quad \text { and } \quad m=\inf _{\bar{\Omega}} W .
$$

Later in 1986, the above estimate of lower bound was improved by Yu-Zhong in [8] via following the similar techniques as [6] and [7] to $\lambda_{2}-\lambda_{1} \geqslant \frac{\pi^{2}}{d^{2}}$. Yu-Zhong's result was thought to be the sharp estimate of lower bound of the gap for the first two eigenvalues under the above assumption. Next we state Yu-Zhong's result asserted in [8] as follows.

Theorem 1.2. ( $\mathbf{Y u}-\mathbf{Z h o n g})$. Let $\Omega$ be a smooth strictly convex bounded domain in $\mathbb{R}^{n}$ and $W: \bar{\Omega} \mapsto \mathbb{R}$ a nonnegative convex function. Suppose $\lambda_{1}$ and $\lambda_{2}$ are the first and second nonzero eigenvalues of (2.1). Then

$$
\lambda_{2}-\lambda_{1} \geqslant \frac{\pi^{2}}{d^{2}}
$$

where $d$ is the diameter of $\Omega$.

In present paper we give another simple proof of Theorem 1.2. Our argument is based on many early works, e.g., Li-Yau [5], Singer-Wong-Yau-Yau [6] and Yu-Zhong [8]. One feature of this argument is that it avoids various kinds of trouble from the singularity of $|\nabla u|^{2} /\left(1-u^{2}\right)$ in those papers. Although our argument many ways analogous to [8], we can readily handle the above singularity, and reduce the difficulty in calculation to a certain extent.

\section{Preliminaries}

Let $\Omega \subset \mathbb{R}^{n}$ be a smooth strictly convex bounded domain and $W: \bar{\Omega} \mapsto \mathbb{R}$ a nonnegative convex smooth function. Consider the following Dirichlet eigenvalue problem of Schrödinger equation

$$
\left\{\begin{array}{l}
-\Delta f+W f=\lambda f \\
f=0 \quad \text { on } \quad \partial \Omega .
\end{array} \text { in } \Omega,\right.
$$

According to a result of [12], the eigenvalues of the Dirichlet problem (2.1) can be arranged in nondecreasing order as follows

$$
0<\lambda_{1}<\lambda_{2} \leqslant \lambda_{3} \leqslant \cdots .
$$


Let $f_{1}$ and $f_{2}$ be the first and second eigenfunctions of (2.1), respectively. It is well known that (e.g. [12]) $f_{1}(x)>0, x \in \Omega$, and $u=f_{2} / f_{1}$ is smooth to the boundary $\partial \Omega$ of $\Omega$ (e.g. [6], p.331). Direct computation implies that

$$
\Delta u+\lambda u+2 \nabla u \cdot \nabla\left(\log f_{1}\right)=0 \quad \text { in } \quad \Omega,
$$

where $\lambda=\lambda_{2}-\lambda_{1}>0$. Clearly, $\log f_{1}$ is well-defined since $f_{1}>0$ on $\Omega$. Without loss of generality, we may assume that

$$
\max u=1, \quad \min u=-k \quad \text { and } \quad 0<k \leqslant 1 .
$$

Set

$$
\left\{\begin{array}{l}
\tilde{u}=\left(u-\frac{1-k}{2}\right) / \frac{1+k}{2} \\
a=\frac{1-k}{1+k}, \quad 0 \leqslant a<1 .
\end{array}\right.
$$

So (2.2) can be rewitten as follows

$$
\left\{\begin{array}{l}
\Delta \tilde{u}+\lambda(\tilde{u}+a)+2 \nabla \tilde{u} \cdot \nabla \log f_{1}=0 \quad \text { in } \quad \Omega \\
\max \tilde{u}=1, \quad \min \tilde{u}=-1 .
\end{array}\right.
$$

Now if we set $\theta=\arcsin \tilde{u}$, Then $\tilde{u}=\sin \theta$ and $-\frac{\pi}{2} \leqslant \theta \leqslant \frac{\pi}{2}$. Define a subset of $\bar{\Omega}$ as follows

$$
\Sigma_{*}=\left\{x \in \bar{\Omega}: \theta(x)=\frac{\pi}{2} \quad \text { or } \quad \theta(x)=-\frac{\pi}{2}\right\} .
$$

By (2.3), a straight forward calculation shows that $\theta$ satisfies

(2.4) $\cos \theta \cdot \Delta \theta-\sin \theta \cdot|\nabla \theta|^{2}+\lambda(\sin \theta+a)+2 \cos \theta \cdot \nabla \theta \cdot \nabla \log f_{1}=0 \quad$ in $\quad \Omega$.

In particular,

$$
\Delta \theta=\frac{\sin \theta}{\cos \theta} \cdot|\nabla \theta|^{2}-\frac{\lambda(\sin \theta+a)}{\cos \theta}-2 \nabla \theta \cdot \nabla \log f_{1} \quad \text { in } \quad \Omega \backslash \Sigma_{*} .
$$

From (2.4), we know that

$$
|\nabla \theta|^{2}=\lambda(1-a) \quad \text { as } \quad \theta=-\frac{\pi}{2},
$$

and

$$
|\nabla \theta|^{2}=\lambda(1+a) \quad \text { as } \quad \theta=\frac{\pi}{2} .
$$

We also define a function $F:\left(-\frac{\pi}{2}, \frac{\pi}{2}\right) \mapsto \mathbb{R}$ as follows

$$
F\left(\theta_{0}\right)=\max _{x \in \Omega, \theta(x)=\theta_{0}}|\nabla \theta(x)|^{2}, \quad \forall \theta_{0} \in\left(-\frac{\pi}{2}, \frac{\pi}{2}\right) .
$$


Obviously, $F$ is well-defined. Actually, $F\left(\theta_{0}\right)$ is not something but an extreme value of $f$ with condition $\theta(x)=\theta_{0}$. It is very easy to verify that $F(\theta)$ is continuous in $\left(-\frac{\pi}{2}, \frac{\pi}{2}\right)$. Moreover, by (2.6) and (2.7), if we define

$$
F\left(-\frac{\pi}{2}\right)=F\left(-\frac{\pi}{2}+0\right)=\lambda(1-a)
$$

and

$$
F\left(\frac{\pi}{2}\right)=F\left(\frac{\pi}{2}-0\right)=\lambda(1+a),
$$

then $F(\theta)$ can be extended a continuous function on $\left[-\frac{\pi}{2}, \frac{\pi}{2}\right]$.

\section{A Rough Estimate of $|\nabla \theta|^{2}$}

Firstly, in a similar way owing to [6, 8] and [10], we get the following lemma.

Lemma 3.1. Let $g(\theta)$ be a smooth function defined on $\left[-\frac{\pi}{2}, \frac{\pi}{2}\right]$. Assume that

$$
G(x)=|\nabla \theta|^{2}+g(\theta(x))
$$

arrives on its maximum at $p \in \partial \Omega \backslash \Sigma_{*}$. Then $\nabla \theta(p)=0$.

Proof. We pick an orthonormal frame $\left\{e_{1}, e_{2}, \cdots, e_{n}\right\}$ around $p$ such that $e_{1}$ is the unit normal of $\partial \Omega$ pointing outward to $\Omega$. We also denote below by $\frac{\partial}{\partial x_{1}}$ the restriction on $\partial \Omega$ of the directional derivative corresponding to $e_{1}$. By the maximality of $G(x)$ at $p$, we also have

$$
0 \leqslant \frac{\partial G(p)}{\partial x_{1}}=2 \sum_{i=1}^{n} \theta_{i}(p) \cdot \theta_{i 1}(p)+g^{\prime}(\theta(p)) \cdot \theta_{1}(p)
$$

In addition, we know by (2.5) that

$$
\nabla \theta \cdot \nabla \log f_{1}=\frac{\nabla \theta \cdot \nabla f_{1}}{f_{1}}=\frac{1}{f_{1}} \sum_{i=1}^{n} \theta_{i} \cdot\left(f_{1}\right)_{i}
$$

achieves finite value on $\partial \Omega \backslash \Sigma_{*}$. But $f_{1}=0$ on $\partial \Omega$, thus

$$
\theta_{1} \cdot\left(f_{1}\right)_{1}+\sum_{i=2}^{n} \theta_{i} \cdot\left(f_{1}\right)_{i}=\sum_{i=1}^{n} \theta_{i} \cdot\left(f_{1}\right)_{i}=0 \quad \text { on } \quad \partial \Omega \backslash \Sigma_{*} .
$$

Since $f_{1} \equiv 0$ on $\partial \Omega$ and $e_{i}(2 \leqslant i \leqslant n)$ are all the tangent vectors of $\partial \Omega$,

$$
\left.\left(f_{1}\right)_{i}\right|_{\partial \Omega}=0 \quad \text { for all } \quad 2 \leqslant i \leqslant n \text {. }
$$


Hence, (3.2) can be reduced to

$$
\theta_{1} \cdot\left(f_{1}\right)_{1}=0 \quad \text { on } \quad \partial \Omega \backslash \Sigma_{*} .
$$

However, Hopf's lemma asserts that $\left(f_{1}\right)_{1}(p)=\frac{\partial f_{1}(p)}{\partial x_{1}} \neq 0$. Therefore,

$$
\theta_{1}(p)=0 \text {. }
$$

Putting (3.3) into (3.1), we then have

$$
0 \leqslant \frac{\partial G(p)}{\partial x_{1}}=2 \sum_{i=2}^{n} \theta_{i}(p) \cdot \theta_{i 1}(p)
$$

Note $\theta_{1}(p)=0$ and recall the definition of second fundamental form of a hypersurface in $\mathbb{R}^{n}$, one can derive

$$
\theta_{i 1}=-\sum_{j=2}^{n} h_{i j} \theta_{j}
$$

where $\left(h_{i j}\right)_{2 \leqslant i, j \leqslant n}$ is the second fundamental form of $\partial \Omega$ relative to $e_{1}$.

It is known that $\Omega$ is strictly convex if and only if $\left(h_{i j}\right)_{2 \leqslant i, j \leqslant n}$ is positive definite. Putting (3.5) into (3.4), we thus have

$$
0 \leqslant \frac{\partial G(p)}{\partial x_{1}}=-2 \sum_{i, j=2}^{n} \theta_{i}(p) h_{i j}(p) \theta_{j}(p) \leqslant 0
$$

Hence, $\theta_{i}(p)=0,2 \leqslant i \leqslant n$. By (3.3) again, we have $\nabla \theta(p)=0$.

As [7] points out that the estimate of the upper bound of $|\nabla \theta|^{2}$ plays an important role in the estimate of the lower bound for $\lambda=\lambda_{2}-\lambda_{1}$. In the following we establish a rough estimate for $|\nabla \theta|^{2}$.

Lemma 3.2. (see [7]) The following estimate is valid

$$
|\nabla \theta(x)|^{2} \leqslant \lambda(1+a), \quad \forall x \in \Omega .
$$

Moreover,

$$
F(\theta) \leqslant \lambda(1+a)
$$

Proof. Suppose that $|\nabla \theta|^{2}$ attains its local maximum at $x_{0} \in \Omega$. Clearly, (2.6) and (2.7) imply that (3.6) holds in the case: $x_{0} \in \Sigma_{*}$. Without loss of generality, we may assume further that $x_{0} \in \bar{\Omega} \backslash \Sigma_{*}$. Thus $\theta_{0}=\theta\left(x_{0}\right) \in\left(-\frac{\pi}{2}, \frac{\pi}{2}\right)$. We easily know from Lemma 3.1 that $\nabla \theta\left(x_{0}\right)=0$ if $x_{0} \in \partial \Omega \backslash \Sigma_{*}$. . Obviously, the conclusion is valid 
in this case. So, we suppose that $x_{0} \in \Omega \backslash \Sigma_{*}$ in the rest of the proof. One compute easily that

$$
\frac{1}{2}\left(|\nabla \theta|^{2}\right)_{j}=\frac{1}{2} \sum_{i}\left(\theta_{i}^{2}\right)_{j}=\sum_{i} \theta_{i} \theta_{i j},
$$

and

$$
\begin{aligned}
\frac{1}{2} \Delta\left(|\nabla \theta|^{2}\right) & =\frac{1}{2} \sum_{j}\left(|\nabla \theta|^{2}\right)_{j j}=\sum_{j}\left(\sum_{i} \theta_{i} \theta_{i j}\right)_{j} \\
& =\sum_{i, j}\left(\theta_{i j}^{2}+\theta_{i} \theta_{i j j}\right)=\left|\nabla^{2} \theta\right|^{2}+\nabla \theta \cdot \nabla(\Delta \theta) .
\end{aligned}
$$

Putting (2.5) into (3.8), we have

$$
\begin{aligned}
\frac{1}{2} \Delta\left(|\nabla \theta|^{2}\right)= & \left|\nabla^{2} \theta\right|^{2}+\nabla \theta \cdot \nabla\left[\frac{\sin \theta}{\cos \theta} \cdot|\nabla \theta|^{2}-\frac{\lambda(\sin \theta+a)}{\cos \theta}\right. \\
& \left.-2 \nabla \theta \cdot \nabla \log f_{1}\right] \\
= & \left|\nabla^{2} \theta\right|^{2}+\nabla \theta \cdot \nabla\left(\frac{\sin \theta}{\cos \theta}\right) \cdot|\nabla \theta|^{2}+\nabla \theta \cdot \frac{\sin \theta}{\cos \theta} \cdot \nabla\left(|\nabla \theta|^{2}\right) \\
& -\lambda \cdot \nabla \theta \cdot\left[\nabla\left(\frac{\sin \theta}{\cos \theta}\right)+a \cdot \nabla\left(\frac{1}{\cos \theta}\right)\right] \\
& -2\left(\nabla \theta \cdot \nabla^{2} \theta\right) \cdot \nabla \log f_{1}-2 \nabla \theta \cdot\left(\nabla \theta \cdot \nabla^{2} \log f_{1}\right) .
\end{aligned}
$$

A direct calculation leads to that

$$
\nabla\left(\frac{\sin \theta}{\cos \theta}\right)=\frac{\nabla(\sin \theta) \cdot \cos \theta-\sin \theta \cdot \nabla(\cos \theta)}{\cos ^{2} \theta}=\frac{\nabla \theta}{\cos ^{2} \theta},
$$

and

$$
\nabla\left(\frac{1}{\cos \theta}\right)=\frac{-1}{\cos ^{2} \theta} \cdot(-\sin \theta) \cdot \nabla \theta=\frac{\sin \theta \cdot \nabla \theta}{\cos ^{2} \theta} .
$$

Putting (3.10) and (3.11) into (3.9), we obtain

$$
\begin{aligned}
\frac{1}{2} \Delta\left(|\nabla \theta|^{2}\right)= & \left|\nabla^{2} \theta\right|^{2}+\frac{|\nabla \theta|^{4}}{\cos ^{2} \theta}+\nabla \theta \cdot \frac{\sin \theta}{\cos \theta} \cdot \nabla\left(|\nabla \theta|^{2}\right) \\
& -\lambda \cdot|\nabla \theta|^{2} \cdot \frac{1+a \sin \theta}{\cos ^{2} \theta}-\nabla\left(|\nabla \theta|^{2}\right) \cdot \nabla \log f_{1} \\
& -2 \nabla \theta \cdot\left(\nabla \theta \cdot \nabla^{2} \log f_{1}\right) .
\end{aligned}
$$

Since $W$ and $\Omega$ are all convex, $\log f_{1}$ is concave by assumption, according to a result of Brascamp and Liep [11], $\log f_{1}$ is concave, i. e., $\left(\nabla^{2} \log f_{1}\right)$ is non-positive definite. Thus

$$
\nabla \theta \cdot\left(\nabla \theta \cdot \nabla^{2} \log f_{1}\right) \leqslant 0
$$


In addition, according to maximum principle, we easily know that at $x_{0}$

$$
\nabla\left(|\nabla \theta|^{2}\right)=0 \quad \text { and } \quad \Delta\left(|\nabla \theta|^{2}\right) \leqslant 0,
$$

Noticing that (3.13) and (3.14), we deduce from (3.12) that at $x_{0}$

$$
0 \geqslant \frac{|\nabla \theta|^{4}}{\cos ^{2} \theta}-\lambda \cdot|\nabla \theta|^{2} \cdot \frac{1+a \sin \theta}{\cos ^{2} \theta} .
$$

Dividing by $|\nabla \theta|^{2}$ and multiplying by $\cos ^{2} \theta$ successively, it follows that at $x_{0}$

$$
0 \geqslant|\nabla \theta|^{2}-\lambda(1+a \sin \theta) .
$$

Hence we have

$$
\left|\nabla \theta\left(x_{0}\right)\right|^{2} \leqslant \lambda\left(1+a \sin \theta_{0}\right) \leqslant \lambda(1+a) .
$$

The proof is complete.

\section{The Estimate of $F(\theta)$}

In the sequel, without loss of generality, we may assume $0<a<1$. In fact, $F(\theta) \leqslant \lambda$ is the best estimate of $F$ when $a=0$. What we want now is get a more precise estimate on $F(\theta)$ than Lemma 3.2. For this purpose, let us introduce the function $\phi(\theta): \Omega \mapsto \mathbb{R}$ such that

$$
F(\theta)=\lambda[1+a \phi(\theta)] .
$$

By Lemma 3.2, it is also easy to see that $\phi(\theta) \leqslant 1$. We shall also need the following technique lemma to estimate accurately $\phi(\theta)$.

Lemma 4.1. (see [7]) Assume that $h:\left[-\frac{\pi}{2},-\frac{\pi}{2}\right] \mapsto \mathbb{R}$ is a nondecreasing function, i.e., $h^{\prime}(\theta) \geqslant 0$, and satisfies

(1) $h(\theta) \geqslant \phi(\theta)$,

(2) there exists some $\theta_{0} \in\left(-\frac{\pi}{2},-\frac{\pi}{2}\right)$, such that $h\left(\theta_{0}\right)=\phi\left(\theta_{0}\right) \geqslant-1$.

Then the following estimate holds

$$
\phi\left(\theta_{0}\right) \leqslant \sin \theta_{0}-\sin \theta_{0} \cdot \cos \theta_{0} \cdot h^{\prime}\left(\theta_{0}\right)+\frac{\cos ^{2} \theta_{0}}{2} \cdot h^{\prime \prime}\left(\theta_{0}\right) .
$$

Proof. Set

$$
\mathcal{E}(x)=\frac{1}{2}\left\{|\nabla \theta(x)|^{2}-\lambda[1+a h(\theta(x))]\right\} .
$$

Obviously, $\mathcal{E}(x) \leqslant 0$ for all $x \in \bar{\Omega}$. By (2.8), we know that there exists some $x_{0} \in \bar{\Omega} \backslash \Sigma_{*}$ such that $\theta\left(x_{0}\right)=\theta_{0}$ and $F\left(\theta_{0}\right)=\left|\nabla \theta\left(x_{0}\right)\right|^{2}$. Thus $\mathcal{E}(x)$ achieves its maximum 0 at $x_{0}$, i. e., 


$$
\left|\nabla \theta\left(x_{0}\right)\right|^{2}=\lambda\left[1+a \phi\left(\theta_{0}\right)\right]=\lambda\left[1+a h\left(\theta_{0}\right)\right] .
$$

Obviously, it is easy to verify that the hypothesis of Lemma 3.1 is satisfied if $2 \mathcal{E}(x)$ is being used in place of $G(x)$. By Lemma 3.1, if $x_{0} \in \partial \Omega \backslash \Sigma_{*}$, then $\nabla \theta\left(x_{0}\right)=0$. Since $0<a<1$ and $\phi\left(\theta_{0}\right) \geqslant-1$,

$$
0=\mathcal{E}\left(x_{0}\right)=\frac{1}{2}\left\{\left|\nabla \theta\left(x_{0}\right)\right|^{2}-\lambda\left[1+a h\left(\theta_{0}\right)\right]\right\} \leqslant-\frac{\lambda(1-a)}{2}<0 .
$$

But this is a contradiction. Hence, $x_{0} \in \Omega \backslash \Sigma_{*}$. It is obvious that at $x_{0}$

$$
\nabla \mathcal{E}=0 \quad \text { and } \quad \Delta \mathcal{E} \leqslant 0,
$$

by maximum principle again. Direct computation shows that

$$
\mathcal{E}_{j}=\sum_{i} \theta_{i} \cdot \theta_{i j}-\frac{\lambda a}{2} h^{\prime}(\theta) \cdot \theta_{j}
$$

namely,

$$
\nabla \mathcal{E}=\frac{1}{2}\left[\nabla\left(|\nabla \theta|^{2}\right)-\lambda a h^{\prime}(\theta) \cdot \nabla \theta\right]=\nabla \theta \cdot \nabla^{2} \theta-\frac{\lambda a}{2} h^{\prime}(\theta) \cdot \nabla \theta .
$$

Since $\nabla \mathcal{E}=0$ at $x_{0}$,

$$
\nabla\left(|\nabla \theta|^{2}\right)=2 \nabla \theta \cdot \nabla^{2} \theta=\lambda a h^{\prime}\left(\theta_{0}\right) \cdot \nabla \theta \quad \text { at } \quad x_{0} .
$$

By directly calculating and applying (2.5), we also obtain

$$
\begin{aligned}
\frac{1}{2} \Delta[\lambda(1+a h)]= & \frac{1}{2} \sum_{j}[\lambda(1+a h)]_{j j}=\frac{\lambda a}{2} \sum_{j}\left(h^{\prime} \cdot \theta_{j}\right)_{j} \\
= & \frac{\lambda a}{2} \sum_{j}\left(h^{\prime \prime} \cdot \theta_{j}^{2}+h^{\prime} \cdot \theta_{j j}\right)=\frac{\lambda a}{2}\left(h^{\prime \prime} \cdot|\nabla \theta|^{2}+h^{\prime} \cdot \Delta \theta\right) \\
= & \frac{\lambda a}{2}\left\{h^{\prime \prime} \cdot|\nabla \theta|^{2}+h^{\prime} \cdot\left[\frac{\sin \theta}{\cos \theta} \cdot|\nabla \theta|^{2}-\frac{\lambda(\sin \theta+a)}{\cos \theta}\right.\right. \\
& \left.\left.-2 \nabla \theta \cdot \nabla \log f_{1}\right]\right\} .
\end{aligned}
$$

Combining (3.12) with (4.6), we hence obtain

$$
\begin{aligned}
\Delta \mathcal{E}= & \left|\nabla^{2} \theta\right|^{2}+\frac{|\nabla \theta|^{4}}{\cos ^{2} \theta}+\nabla \theta \cdot \frac{\sin \theta}{\cos \theta} \cdot \nabla\left(|\nabla \theta|^{2}\right) \\
& -\lambda \cdot|\nabla \theta|^{2} \cdot \frac{1+a \sin \theta}{\cos ^{2} \theta}-2\left(\nabla \theta \cdot \nabla^{2} \theta\right) \cdot \nabla \log f_{1} \\
& -2 \nabla \theta \cdot\left(\nabla \theta \cdot \nabla^{2} \log f_{1}\right)-\frac{\lambda a}{2}\left\{h^{\prime \prime} \cdot|\nabla \theta|^{2}\right. \\
& \left.+h^{\prime} \cdot\left[\frac{\sin \theta}{\cos \theta} \cdot|\nabla \theta|^{2}-\frac{\lambda(\sin \theta+a)}{\cos \theta}-2 \nabla \theta \cdot \nabla \log f_{1}\right]\right\} .
\end{aligned}
$$


Inserting (4.5) into (4.7), it is easy to deduce that at $x_{0}$

$$
\begin{aligned}
\Delta \mathcal{E}= & \left|\nabla^{2} \theta\right|^{2}+\frac{|\nabla \theta|^{4}}{\cos ^{2} \theta}+\lambda a h^{\prime} \cdot \frac{\sin \theta}{\cos \theta} \cdot|\nabla \theta|^{2} \\
& -\lambda \cdot|\nabla \theta|^{2} \cdot \frac{1+a \sin \theta}{\cos ^{2} \theta}-\lambda a h^{\prime} \cdot \nabla \theta \cdot \nabla \log f_{1} \\
& -2 \nabla \theta \cdot\left(\nabla \theta \cdot \nabla^{2} \log f_{1}\right)-\frac{\lambda a}{2}\left\{h^{\prime \prime} \cdot|\nabla \theta|^{2}\right. \\
& \left.+h^{\prime} \cdot\left[\frac{\sin \theta}{\cos \theta} \cdot|\nabla \theta|^{2}-\frac{\lambda(\sin \theta+a)}{\cos \theta}-2 \nabla \theta \cdot \nabla \log f_{1}\right]\right\} .
\end{aligned}
$$

Rearranging the terms, the above equality reduces to

$$
\begin{aligned}
\Delta \mathcal{E}= & \left|\nabla^{2} \theta\right|^{2}+\frac{|\nabla \theta|^{4}}{\cos ^{2} \theta}+\lambda a h^{\prime} \cdot \frac{\sin \theta}{\cos \theta} \cdot|\nabla \theta|^{2} \\
& -\lambda \cdot|\nabla \theta|^{2} \cdot \frac{1+a \sin \theta}{\cos ^{2} \theta}-2 \nabla \theta \cdot\left(\nabla \theta \cdot \nabla^{2} \log f_{1}\right) \\
& -\frac{\lambda a}{2}\left\{h^{\prime \prime} \cdot|\nabla \theta|^{2}+h^{\prime} \cdot\left[\frac{\sin \theta}{\cos \theta} \cdot|\nabla \theta|^{2}-\frac{\lambda(\sin \theta+a)}{\cos \theta}\right]\right\} .
\end{aligned}
$$

By virtue of (4.3) and (4.4), we derive from (4.8) that at $x_{0}$

$$
\begin{aligned}
0 \geqslant & \left|\nabla^{2} \theta\right|^{2}+\frac{\lambda^{2}(1+a h)^{2}}{\cos ^{2} \theta}+\lambda^{2} a h^{\prime}(1+a h) \frac{\sin \theta}{\cos \theta} \\
& -\lambda^{2}(1+a h) \frac{1+a \sin \theta}{\cos ^{2} \theta}-2 \nabla \theta \cdot\left(\nabla \theta \cdot \nabla^{2} \log f_{1}\right) \\
& -\frac{\lambda a}{2}\left\{h^{\prime \prime} \cdot|\nabla \theta|^{2}+h^{\prime} \cdot\left[\frac{\sin \theta}{\cos \theta} \cdot|\nabla \theta|^{2}-\frac{\lambda(\sin \theta+a)}{\cos \theta}\right]\right\} \\
= & \left|\nabla^{2} \theta\right|^{2}+\frac{\lambda^{2}(1+a h)^{2}}{\cos ^{2} \theta}-\lambda^{2}(1+a h) \frac{1+a \sin \theta}{\cos ^{2} \theta} \\
& -2 \nabla \theta \cdot\left(\nabla \theta \cdot \nabla^{2} \log f_{1}\right)+\frac{\lambda^{2} a}{2}\left\{-h^{\prime \prime}(1+a h)\right. \\
& \left.+h^{\prime} \cdot\left[\frac{\sin \theta}{\cos \theta}(1+a h)+\frac{(\sin \theta+a)}{\cos \theta}\right]\right\} .
\end{aligned}
$$

Obviously, we easily know by (3.13) that the first term and the fourth term on the right-hand side can be dropped because they are nonnegative. After dividing by $\lambda^{2} a$, multiplying by $\cos ^{2} \theta$ and rearranging the terms successively, we are led to 


$$
\begin{aligned}
0 \geqslant & \frac{(1+a h)^{2}}{a}-\frac{(1+a h)(1+a \sin \theta)}{a}-\frac{h^{\prime \prime}(1+a h) \cos ^{2} \theta}{2} \\
& +\frac{h^{\prime}(1+a h) \cos \theta \sin \theta}{2}+\frac{h^{\prime} \cos \theta(\sin \theta+a)}{2} \\
= & (1+a h)(h-\sin \theta)-\frac{h^{\prime \prime}(1+a h) \cos ^{2} \theta}{2} \\
& +\frac{h^{\prime} \cos \theta}{2}[(1+a h) \sin \theta+(\sin \theta+a)] .
\end{aligned}
$$

Since $h\left(\theta_{0}\right)=\phi\left(\theta_{0}\right) \geqslant-1$ and $\phi\left(\theta_{0}\right)=\phi\left(\theta\left(x_{0}\right)\right) \leqslant 1$, then $\left|h\left(\theta_{0}\right)\right| \leqslant 1$.

From $|h|=|h(\theta)| \leqslant 1$ at $x_{0}$, and $0<a<1$, it follows that at $x_{0}$

$$
a \geqslant a h \sin \theta \quad \text { and } \quad 1+a h>0 .
$$

Thus, at $x_{0}$

$$
\sin \theta+a \geqslant \sin \theta+a h \sin \theta=(1+a h) \sin \theta
$$

Hence, under the assumption that $h^{\prime}(\theta) \geqslant 0$, using (4.11), we proceed by tackling with (4.10) at $x_{0}$ as follows

$$
0 \geqslant(1+a h)(h-\sin \theta)+h^{\prime}(1+a h) \cos \theta \sin \theta-\frac{h^{\prime \prime}(1+a h) \cos ^{2} \theta}{2} .
$$

Dividing by $1+a h$, we have at $x_{0}$

$$
0 \geqslant(h-\sin \theta)+h^{\prime} \cos \theta \sin \theta-\frac{h^{\prime \prime} \cos ^{2} \theta}{2} .
$$

Obviously, (4.2) follows from (4.12) immediately.

The remaining part of the present paper works exactly as in [7] and [8]. For the completeness we briefly sketch a proof of Theorem 1.2 below which only use the methods owing to [7] and [8]. We refer the reader to consult [7], [8] and [10] for more details.

Lemma 4.2. (see [7]) Define function $\psi$ as follows

$$
\left\{\begin{array}{l}
\psi(\theta)=\frac{\frac{4}{\pi}(\theta+\cos \theta \sin \theta)-2 \sin \theta}{\cos ^{2} \theta}, \quad \theta \in\left(-\frac{\pi}{2}, \frac{\pi}{2}\right), \\
\psi\left(-\frac{\pi}{2}\right)=-1, \quad \psi\left(\frac{\pi}{2}\right)=1 .
\end{array}\right.
$$

Then $\psi \in C^{0}\left[-\frac{\pi}{2}, \frac{\pi}{2}\right] \cap C^{2}\left(-\frac{\pi}{2}, \frac{\pi}{2}\right)$, satisfies $\psi^{\prime}(\theta) \geqslant 0$ and

$$
\psi(\theta)-\sin \theta+\sin \theta \cdot \cos \theta \cdot \psi^{\prime}(\theta)-\frac{\cos ^{2} \theta}{2} \cdot \psi^{\prime \prime}(\theta)=0 .
$$

Moreover, the following properties

$$
|\psi(\theta)| \leqslant 1 \quad \text { and } \quad \psi(-\theta)=-\psi(\theta) .
$$

hold for any $\theta \in\left[-\frac{\pi}{2}, \frac{\pi}{2}\right]$. 
Using Lemma 4.1, Lemma 4.2 and the method of proof by contradiction, we easily deduce the following lemma. For convenience of the reader, we give a proof below which is due to Zhong-Yang [7].

Lemma 4.3. (see [7]). Assume that $\phi(\theta)$ and $\psi(\theta)$ are defined by (4.1) and (4.13), respectively. Then

$$
\phi(\theta) \leqslant \psi(\theta) .
$$

Proof. Assume that (4.15) is not true. Since $\phi\left( \pm \frac{\pi}{2}\right)= \pm 1=\psi\left( \pm \frac{\pi}{2}\right)$, then there exists some $\theta_{0} \in\left(-\frac{\pi}{2}, \frac{\pi}{2}\right)$ such that

$$
\sigma=\phi\left(\theta_{0}\right)-\psi\left(\theta_{0}\right)=\max _{-\frac{\pi}{2} \leqslant \theta \leqslant \frac{\pi}{2}}\{\phi(\theta)-\psi(\theta)\}>0 .
$$

Set $\widetilde{h}(\theta)=\psi(\theta)+\sigma$. Obviously, $\widetilde{h}^{\prime}(\theta)=\psi^{\prime}(\theta) \geqslant 0$,

$$
\widetilde{h}(\theta)=\psi(\theta)+\sigma \geqslant \phi(\theta)
$$

and

$$
\widetilde{h}\left(\theta_{0}\right)=\phi\left(\theta_{0}\right)=\underset{\sim}{\psi}\left(\theta_{0}\right)+\sigma \geqslant-1+\sigma>-1 .
$$

In place of $h(\theta)$ in Lemma 4.1 by $\widetilde{h}(\theta)$, we therefore get by Lemma 4.1 and Lemma 4.2 that

$$
\begin{aligned}
\phi\left(\theta_{0}\right) & \leqslant \sin \theta_{0}-\sin \theta_{0} \cdot \cos \theta_{0} \cdot \widetilde{h}^{\prime}\left(\theta_{0}\right)+\frac{\cos ^{2} \theta_{0}}{2} \cdot \widetilde{h}^{\prime \prime}\left(\theta_{0}\right) \\
& =\sin \theta_{0}-\sin \theta_{0} \cdot \cos \theta_{0} \cdot \psi^{\prime}\left(\theta_{0}\right)+\frac{\cos ^{2} \theta_{0}}{2} \cdot \psi^{\prime \prime}\left(\theta_{0}\right)=\psi\left(\theta_{0}\right) .
\end{aligned}
$$

But this contradicts (4.16).

Corollary 4.1. (see [7]). The following estimate holds.

$$
F(\theta) \leqslant \lambda[1+a \psi(\theta)]
$$

where $F(\theta)$ and $\psi(\theta)$ are defined by (2.8) and (4.13), respectively.

Our argument above establishes the inequality (4.17), which is the refined estimate of the upper bound for $F(\theta)$ as required.

\section{Proof of Theorem 1.2}

We stress here that although a reasoning similar to the one in [7] and [8] will give the claim, our proof is slightly different from [7] , [8] and [10]. We now use the estimate of $F(\theta)$ to prove Theorem 1.2 in the following.

Proof. (4.17) implies that 


$$
\lambda^{\frac{1}{2}} \geqslant \frac{|F(\theta)|^{\frac{1}{2}}}{\sqrt{1+a \psi(\theta)}} \geqslant \frac{|\nabla \theta|}{\sqrt{1+a \psi(\theta)}},
$$

where $\psi(\theta)$ is defined by (4.13).

Take $x_{1}, x_{2} \in \Omega$ such that $\theta\left(x_{1}\right)=-\frac{\pi}{2}, \theta\left(x_{2}\right)=\frac{\pi}{2}$. We denote by $d^{\prime}$ the length of the straight line $\gamma$ joining $x_{1}$ and $x_{2}$. Obviously, $d^{\prime} \leqslant d$.

Using (4.14) and the the following inequality of analysis

$$
\frac{1}{\sqrt{1-x}}+\frac{1}{\sqrt{1+x}} \geqslant 2, \quad \forall x \in(-1,1),
$$

we derive from integrating (5.1) along the straight line $\gamma$ that

$$
\begin{aligned}
\lambda^{\frac{1}{2}} d & \geqslant \lambda^{\frac{1}{2}} d^{\prime}=\int_{\gamma} \lambda^{\frac{1}{2}} \mathrm{~d} s \geqslant \int_{\gamma} \frac{1}{\sqrt{1+a \psi(\theta)}}|\nabla \theta| \mathrm{d} s \\
& \geqslant \int_{\gamma} \frac{1}{\sqrt{1+a \psi(\theta)}} \mathrm{d} \theta=\int_{-\frac{\pi}{2}}^{\frac{\pi}{2}} \frac{1}{\sqrt{1+a \psi(\theta)}} \mathrm{d} \theta \\
& =\int_{0}^{\frac{\pi}{2}}\left[\frac{1}{\sqrt{1-a \psi(\theta)}}+\frac{1}{\sqrt{1+a \psi(\theta)}}\right] \mathrm{d} \theta \\
& \geqslant \int_{0}^{\frac{\pi}{2}} 2 \mathrm{~d} \theta=\pi .
\end{aligned}
$$

This concludes the proof of the theorem.

\section{ACKNOWLEDGMENT}

The author wishes to take this opportunity to thank the referee for his (or her) valuable advice and suggestion.

\section{REFERENCES}

1. J. Cheeger, A lower bound for the smallest eigenvalue of the Laplacian, Problems in Analysis, a symposium in honor of S. Bochner, Princeton Univ. Press, Princeton, 1970.

2. P. Li, A lower bound for the first eigenvalue of the Laplacian on a compact Riemannian manifold, Indiana Univ. Math. J., 28 (1979), 1013-1019.

3. P. Li and S.-T. Yau, Eigenvalues of a compact Riemannian manifold, AMS Proc. Symp. Pure Math., 36 (1980), 205-239.

4. P. Li, Survey on partial differential equations in differential geometry, Ann. of Math. Study, 102 (1982), 3-73.

5. P. Li and S.-T. Yau, On the Schrodinger equation and the eigenvalue problem, Comm. Math. Phys., 88(3) (1983), 309-318. 
6. I. M. Singer, B. Wong, S.-T. Yau and Stephen S.-T. Yau, An estimate of the gap of the first two eigenvalues in the Schrodinger operator, Ann. Scuola Norm. Sup. Pisa Cl. Sci. (4), 12(2) (1985), 319-333.

7. J.-Q. Zhong and H.-C. Yang, Estimates of the first eigenvalue of Laplace opeator on compact Riemannian manifolds (in Chinese), Sci. Sinica Ser. A, 9(9) (1983), 812-820. Translated into English: On the estimate of the first eigenvalue of a compact Riemannian manifold, Sci. Sinica Ser. A, 27(12) (1984), 1265-1273.

8. Q.-H. Yu and J.-Q. Zhong, Lower bounds of the gap between the first and second eigenvalues of the Schrödinger operator, Trans. Amer. Math. Soc., 294(1) (1986), 341349.

9. P. Li and S.-T. Yau, On the parabolic kernel of the Schrödinger operator, Acta Math., 156(3-4) (1986), 153-201.

10. R. Schoen and S.-T. Yau, Lectures on Differential Geometry, Conference Proceedings and Lecture Notes in Geometry and Topology, Vol. I, International Press, 1994.

11. H. Brascamp and E. Liep, On extensions of the Brunn-Minkowski and Prékopa-Leindler theorems, including inequalities for log concave function, and with an application to diffusion equation, J. Funct. Anal., 22 (1976), 366-389.

12. R. Courant and D. Hilbert, Methods of mathematical physics, Vol. I, Interscience, New York, 1953.

Yue He

Institute of Mathematics

School of Mathematics Sciences

Nanjing Normal University

Nanjing 210046

P. R. China

E-mail: heyueyn@163.com

heyue@njnu.edu.cn 